\title{
Equations of the Korteweg-de Vries type with non-trivial conserved quantities
}

\author{
Rafael D Benguria† and M Cristina Depassierł \\ † Departamento de Física, F.C.F.M., Universidad de Chile, Casilla 487-3, Santiago, Chile \\ ¥ Facultad de Física, P. Universidad Católica de Chile, Casilla 6177, Santiago 22, Chile
}

Received 8 February 1989

\begin{abstract}
We study a family of equations of the Korteweg-de Vries type. Different elements of this family are characterised by their linear dispersion relation $w(k)$. We prove that there are only three members of this family which possess a non-trivial polynomial conserved quantity and have a linear dispersion relation analytic in a neighbourhood of the origin. These correspond to the Korteweg-de Vries equation itself, the Benjamin-Ono equation and the intermediate long wave equation. As is well known, these equations have indeed infinitely many conserved quantities.
\end{abstract}

\section{Introduction}

One of the remarkable properties of the Korteweg-de Vries (KdV) equation is that it has infinitely many conserved quantities (see, e.g., Miura et al 1968). Typically, autonomous evolution equations with translation invariance have only three conserved quantities, namely the mass, the momentum and the energy. These are the 'classical' conserved quantities. The fact that the KdV equation has infinitely many conserved quantities is directly related to its complete integrability. So far two other equations of the $\mathrm{KdV}$ type with infinitely many conserved quantitites have been found. These are the Benjamin-Ono equation (Benjamin 1967, Ono 1975), and the intermediate long wave (ILW) equation (Joseph 1977, Kubota et al 1978). A natural question we may ask is whether there exist other equations of the same form as the KdV equation which have infinitely many conserved quantities and are, therefore, likely to be completely integrable. This question was considered by Satsuma et al (1979) who, based on the existence of a Bäcklund transformation, gave a criterion (the so-called $T$ conditions) for the existence of infinitely many conserved quantities (see also Ablowitz et al 1982). In this paper we consider a more modest question, namely we search for equations of the KdV type with one non-trivial (meaning non-classical) polynomial conserved quantity. By equations of the KdV type we mean evolution equations having the same non-linear structure as the $\mathrm{KdV}$ equation but different dispersion relations. We find a necessary condition on the dispersion relation (see equation (26) below) in order for the associated evolution equation to have a non-trivial conserved quantity. This equation is more general than the $T$ conditions. Then we show that there are only three solutions to this equation (26) which are analytic in the neighbourhood of $k=0$. These correspond to the dispersion relations for the $\mathrm{KdV}$ equation, for the Benjamin-Ono equation and for the ILW equation. We point out that there are other, non-analytic, solutions to equation (26). 
The rest of this paper is organised as follows. In $\S 2$ we introduce the equations of the KdV type and we find the necessary condition on the dispersion relation in order to have non-trivial conserved quantities. Finally, in $\S 3$ we discuss the existence and multiplicity of solutions to this necessary condition.

\section{Equations of the KdV type}

By equations of the KdV type we mean evolution equations of the form

$$
u_{t}=\frac{\partial}{\partial x}\left(u^{2}+G\left[u_{x}\right]\right)
$$

defined on the whole real line and for $t>0$. Here $u=u(x, t)$, and we use subscripts to denote partial derivatives. In (1), $G$ is the pseudo-differential operator given by

$$
G[f](x) \equiv \int_{-\infty}^{\infty} e^{i k x} \hat{g}(k) \hat{f}(k) d k
$$

where $\hat{f}(k)$ denotes the Fourier transform of $f(x)$ and $\hat{g}(k)$ is the symbol of $G$. We call (1), (2), the family of equations of the KdV type. The symbol $\hat{g}(k)$ determines the dispersion relation of the linearised equation obtained from (1). If $G$ is antisymmetric with respect to the usual inner product $(f, g)=\int_{-\infty}^{\infty} f g \mathrm{~d} x$ (i.e. if $\hat{g}$ is odd as a function of $k$ ) equations (1) and (2) have three 'classical' conserved quantities, namely

$$
\begin{array}{ll}
M=\int_{-\infty}^{\infty} u(x, t) \mathrm{d} x & \text { the mass } \\
P=\int_{-\infty}^{\infty} u^{2}(x, t) \mathrm{d} x & \text { the momentum } \\
E=\int_{-\infty}^{\infty}\left(\frac{1}{3} u^{3}+\frac{1}{2} u G\left[u_{x}\right]\right) \mathrm{d} x & \text { the energy. }
\end{array}
$$

For the Korteweg-de Vries equation, the symbol is given by

$$
\hat{g}(k)=i k
$$

whereas for the Benjamin-Ono equation this is

$$
\hat{g}(k)=\mathrm{i} k /|k|
$$

and for the intermediate long wave equation (ILW)

$$
\hat{g}(k)=\frac{c_{0}}{2 \pi}\left(1-\frac{1}{2} k d \operatorname{coth}(k D)\right) / k
$$

where $c_{0}, d$ and $D$ are free parameters. For the equations corresponding to these three symbols, (1) and (2) have non-classical conserved quantities; in fact they have infinitely many (see, e.g., Miura et al 1968, Case 1979, Satsuma et al 1979, respectively). Are there other symbols $\hat{g}(k)$ for which $(1),(2)$ has at least one non-classical conserved quantity? Here we address a simpler problem: namely, are there other symbols for which (1), (2) has a polynomial conserved quantity of degree four, i.e. of the form

$$
I_{4}=\int_{-\infty}^{\infty}\left(\frac{1}{4} u^{4}+\ldots\right) \mathrm{d} x
$$


As is well known (see, e.g., Gardner 1971, Faddeev et al 1971 or Lax 1975), equations of the form (1), (2) have a Hamiltonian structure. One can write (1) as

$$
u_{t}=\{u, \mathscr{H}\}
$$

where, as usual, the Poisson bracket between two functionals $\mathscr{F}_{1}$ and $\mathscr{F}_{2}$ of $u$ is defined as

$$
\left\{\mathscr{F}_{1}, \mathscr{F}_{2}\right\}=\int_{-\infty}^{\infty} \frac{\delta \mathscr{F}_{1}}{\delta u} \frac{\mathrm{d}}{\mathrm{d} x}\left(\frac{\delta \mathscr{F}_{2}}{\delta u}\right) \mathrm{d} x
$$

where $\delta \mathscr{F} / \delta u$ denotes the functional derivative of $\mathscr{F}$ with respect to $u$. In equation (10), the Hamiltonian $\mathscr{H}$ is just the energy, given by (5). Hence,

$$
\frac{\delta \mathscr{H}}{\delta u}=u^{2}+G\left[u_{x}\right]
$$

and, therefore,

$$
\{u, \mathscr{H}\}=\frac{\partial}{\partial x}\left(u^{2}+G\left[u_{x}\right]\right) .
$$

Because of the Hamiltonian structure, $I_{4}$ is a conserved quantity if and only if

$$
\left\{\mathscr{H}, I_{4}\right\}=0 \text {. }
$$

Let

$$
A_{4} \equiv \frac{\delta I_{4}}{\delta u}=u^{3}+\ldots
$$

denote the functional derivative of $I_{4}$. Our problem reduces to determining whether there is an $A_{4}$ of the form (15) such that

$$
\int_{-\infty}^{\infty}\left(u^{2}+G\left[u_{x}\right]\right)_{x} A_{4} \mathrm{~d} x=0 .
$$

Since $A_{4}=\delta I_{4} / \delta u$ we must require also that

$$
\frac{\delta A_{4}(x)}{\delta u(y)}=\frac{\delta A_{4}(y)}{\delta u(x)} .
$$

The above equation is just the integrability condition for $A_{4}$.

The most general form $A_{4}$ can have, in order to satisfy (15), (17) and (16), is

$$
A_{4}=u^{3}+\frac{3}{2} u G\left[u_{x}\right]+\frac{3}{2} G\left[u u_{x}\right]+K\left[u_{x}\right]
$$

where $K$ is an antisymmetric (i.e. with a symbol odd in $k$ ) pseudo-differential operator. In principle, we could allow the pseudo-differential operator $G$ in (18) to be different than the $G$ given in (2). However, one soon realises that the symbols of both operators have to be the same in order to satisfy (16). In fact, replacing $A_{4}$, given by (18), in equation (16) we get,

$$
\int_{-\infty}^{\infty}\left(\left(u^{2}\right)_{x} M\left[u_{x}\right]+\frac{3}{2} u G\left[u_{x}\right] G\left[u_{x x}\right]\right) \mathrm{d} x=0
$$

with

$$
M[f] \equiv K[f]-\frac{3}{4} G\left[G\left[f_{x}\right]\right]
$$


Therefore, our original question can be rephrased in the following way. 'For what functions $G$ does there exist an $M$ such that (19) holds for all $u$ ?' It is worth pointing out here that (19) is certainly satisfied if $G$ satisfies the so-called $T$ conditions (see Satsuma et al 1979, Ablowitz et al 1982).

Let $\hat{u}(k)$ denote the Fourier transform of $u$, and $\hat{g}(k), \hat{h}(k)$ and $\hat{m}(k)$ the symbols of the pseudo-differential operators $G, K$, and $M$, respectively. In terms of $\hat{u}, \hat{g}, \hat{h}$ and $\hat{m}$, equation (19) becomes

$\int_{-\infty}^{\infty} \int_{-\infty}^{\infty} \hat{u}(k) \hat{u}(q) \hat{u}(-k-q)[\lambda k q(k+q) \hat{g}(k) \hat{g}(q)+\hat{m}(k) k q] \mathrm{d} k \mathrm{~d} q=0$.

Here, $\lambda=3 \mathrm{i} / 8$ is a constant. A necessary and sufficient condition on $\hat{g}$ and $\hat{m}$ so that (21) holds for all $\hat{u}$ is

$$
\lambda k q(k+q) \hat{g}(k) \hat{g}(q)+\hat{m}(k) k q=b(k, q)+d(q,-k-q)+f(-k-q, k)+s(k, q,-k-q)
$$

where $b(\cdot, \cdot), d(\cdot, \cdot)$ and $f(\cdot, \cdot)$ are arbitrary antisymmetric functions of their arguments (i.e. $b(k, q)=-b(q, k)$, etc) and $s(\cdot, \cdot, \cdot)$ is an arbitrary completely antisymmetric function. We can easily get rid of $s$ by changing $b, d$ and $f$ into $\tilde{b}, \tilde{d}$ and $\tilde{f}$ respectively, where $b(k, q)=\tilde{b}(k, q)+s(k, q,-k-q), \quad d(k, q)=$ $\tilde{d}(k, q)-s(k, q,-k-q)$, and $f(k, q)=\tilde{f}(k, q)-s(k, q,-k-q)$. The form of equation (22) imposes strong conditions on $\hat{g}(k)$. In fact, as we shall see in the following, one can obtain an equation involving only $\hat{g}(k)$ (see equation (26), below) which is a necessary condition on $\hat{g}(k)$ so that $(22)$ is satisfied. We proceed as follows.

Step 1. Consider the three equations obtained from (22) by setting respectively $k=l$, $q=-2 l ; k=-2 l, q=l$; and $k=l, q=l$. By adding the three resulting equations we get

$$
\hat{m}(q)-2 \hat{m}(2 q)=2 \lambda q \hat{g}(q)(\hat{g}(q)-2 \hat{g}(2 q))
$$

where we have substituted $l$ by $q$ as the independent variable. In deriving (23) we have used the antisymmetry of $b, d, f$ and $s$.

Step 2. Consider now the six equations obtained from (22) by setting respectively $k=2 l, q=l ; q=2 l, k=l ; q=-3 l, k=l ; q=l, k=-3 l ; q=-3 l, k=2 l ;$ and $q=2 l$, $k=-3 l$. Then, adding the six resulting equations we get

$9 \hat{m}(3 q)-4 \hat{m}(2 q)-\hat{m}(q)=12 \lambda q(-\hat{g}(q) \hat{g}(2 q)+\hat{g}(q) \hat{g}(3 q)+\hat{g}(2 q) \hat{g}(3 q))$

where we have substituted $l$ by $q$ as the independent variable.

Step 3. Finally consider the six equations obtained from (22) by setting respectively $k=3 l, q=l ; k=l, q=3 l ; k=-4 l, q=l ; q=-4 l, k=l ; k=-4 l, q=3 l ;$ and $k=3 l$, $q=-4 l$. Then adding the six resulting equations we get

$16 \hat{m}(4 q)-9 \hat{m}(3 q)-\hat{m}(q)=24 \lambda q(\hat{g}(4 q) \hat{g}(q)-\hat{g}(3 q) \hat{g}(q)+\hat{g}(4 q) \hat{g}(3 q))$

where we have substituted again $l$ by $q$ as the independent variable.

The left-hand sides of equations (23), (24) and (25) are all linear in $\hat{m}$, and it is rather simple to eliminate $\hat{m}$ from these three equations. By doing so we finally get one equation for the symbol $\hat{g}$ of $G$, which is a necessary condition on equation (1) for it to have a non-classical polynomial conserved quantity. The equation we get for $\hat{g}$ is

$$
(3 \hat{g}(3 q)-\hat{g}(q))(\theta(2 q)+\theta(q))=4 \theta(2 q) \theta(q)
$$


where we have introduced

$$
\theta(q) \equiv 2 \hat{g}(2 q)-\hat{g}(q)
$$

Properties of equation (26). It is rather straightforward to verify that the equation we have found for $\hat{g}(q)$ (i.e. equation (26)) has the following properties.

(i) If $\hat{g}(q)$ solves (26), so does $\alpha \hat{g}(\gamma q)+\beta / q$ for any $\alpha, \beta$ and $\gamma$.

(ii) $\hat{g}(q)=q$ (the symbol corresponding to the KdV equation), $\hat{g}(q)=c_{0}$, for $q \geqslant 0$ (the symbol corresponding to the Benjamin-Ono equation), and $\hat{g}(q)=\operatorname{coth}(q)-1 / q$, for $q \geqslant 0$ (the symbol corresponding to the ILw equation) are all analytic solutions of (26) in a neighbourhood of the origin. (Note that here it is enough to consider $\hat{g}(q)$ defined in $[0, \infty)$. Recall that $\hat{g}(-q)=-\hat{g}(q)$.)

(iii) There are many other solutions of (26) which are not analytic near 0 . For example

$$
\hat{g}(q)=\frac{1}{q} \sin \left(2 \pi \frac{\ln (q)}{\ln (2)}\right)
$$

solves (26), and so does any other function satisfying $\hat{g}(2 q)=\frac{1}{2} \hat{g}(q)$ i.e. $\left.\theta(q)=0\right)$. At this point it is worth recalling that $(26)$ is only a necessary condition on $\hat{g}$ for equation (1) to have a non-classical conserved quantity.

\section{Analytic solutions of the equation for $\hat{g}$.}

In this section we find all the solutions of equation (26) which are analytic in a neighbourhood of $q=0$. Let

$$
\hat{\mathbf{g}}(q)=\sum_{n=0}^{\infty} c_{n} q^{n}
$$

be the power series for $\hat{g}(q)$ around $q=0$. Introducing this expression for $\hat{g}(q)$ in equation (26) we get

$$
\sum_{n=0}^{\infty} \omega_{n} q^{n}=0
$$

for all $q>0$ in a neighbourhood of the origin and, therefore,

$$
\omega_{n}=0 \quad \text { for } n=0,1,2, \ldots
$$

where

$$
\omega_{n}= \begin{cases}d_{n} c_{n / 2}^{2}+\sum_{l=0}^{(n / 2)-1} c_{l} c_{n-l} A_{l n} & \text { for } n=2,4, \ldots \\ \sum_{l=0}^{(n-1) / 2} c_{l} c_{n-l} A_{l n} & \text { for } n=1,3,5, \ldots\end{cases}
$$

and $\omega_{0} \equiv 0$. Here,

$$
A_{l n}=B_{l n}+B_{n-l n} \quad \text { for } l<n / 2
$$

with

$B_{l n}=6 \times 4^{l}-3^{l+1}+6 \times 4^{l} \times 3^{n-l}+3^{l+1} 2^{n-l}-5 \times 2^{l}-16 \times 4^{l} \times 2^{n-l}+8 \times 2^{n}+1$ 
and

$$
d_{n}=14 \times 2^{n}+3^{n / 2}\left(6 \times 2^{n}+3 \times 2^{n / 2}-3\right)-5 \times 2^{n / 2}+1-16 \times 2^{3 n / 2} .
$$

Equation (30) defines a recurrence relation for the coefficients $c_{n}$ which assumes the general form

$$
c_{0} c_{n} A_{0 n}=f_{n}\left(c_{1}, c_{2}, \ldots, c_{n-1}\right) .
$$

Denote $b_{n}=A_{0 n}=2\left[3^{n+1}-2 \times 4^{n}-2^{n}\right]$. Clearly $b_{0}=0$ and $b_{n+1}=4 b_{n}+2\left(2^{n+1}-3^{n+1}\right)$. Therefore, $b_{n}<0$ for all $n \geqslant 1$. Now, we divide the study of the recurrence relation (36) (or (30)) into three separate cases.

Case $1, c_{0} \neq 0$. We will show that in this case $c_{n}=0$ for all $n \geqslant 1$. If $n=1,(36)$ is

$$
c_{0} c_{1} b_{1}=0
$$

whereas, for $n \geqslant 2,(36)$ is given by

$$
c_{0} c_{n} b_{n}=f_{n}\left(c_{1}, c_{2}, \ldots, c_{n-1}\right)
$$

where $f_{n}$ is a homogeneous function of degree 2. Since $b_{n} \neq 0$ for all $n \geqslant 1$ (in fact $\left.b_{n}<0\right),(37)$ implies $c_{1}=0$ and, therefore, from (38) we conclude inductively that $c_{n}=0$ for all $n \geqslant 2$. Therefore, in this case the only analytic solution to equation (26) is

$$
\hat{g}(q)=c_{0}
$$

which is the symbol associated with the Benjamin-Ono equation in equation (1).

Case 2, $c_{0}=c_{1}=0$. We will show that in this case $c_{n}=0$ for all $n \geqslant 2$ and, therefore, $\hat{g} \equiv 0$. From (31) we have that $\omega_{4}=0$ implies

$$
d_{4} c_{2}^{2}=0
$$

whereas for $n$ even, $n \geqslant 6$, we have from (31) that

$$
\sum_{i=2}^{(n / 2)-1} c_{l} c_{n-l} A_{l n}+d_{n} c_{n / 2}^{2}=0 .
$$

From (35) we have that $d_{4}=126$ and therefore (40) implies $c_{2}=0$. Moreover, $d_{n}>0$ for all $n \geqslant 6$. In fact, after some regrouping we get from (35) that

$$
d_{n}=2^{n / 2} \sigma_{n}+3^{(n / 2)+1} \tau_{n}+2^{3 n / 2} \theta_{n}
$$

with

$$
\sigma_{n}=14 \times 2^{n / 2}-5 \quad \tau_{n}=2^{n / 2}-1 \quad \theta_{n}=(3 / 2)^{n / 2} 6-16 .
$$

It is clear that $\sigma_{n}>0, \tau_{n}>0$ for all $n>0$. Moreover, $\theta_{n}>0$ for $n \geqslant 6$. Thus, $d_{n}>0$ for all $n \geqslant 6$. (In fact, $d_{n}>0$ for $n=2,4, \ldots$ ) Since $d_{n} \neq 0$ for all $n \geqslant 6, n$ even, and $c_{2}=0$, it follows inductively from (41) that $c_{n}=0$ for all $n$. This also ensures that $\omega_{n}=0$ is satisfied when $n$ is odd.

Case 3, $c_{0}=0, c_{1} \neq 0$. If $c_{0}=0$ we get from (31) and (32) that the equation $\omega_{n}=0$ reduces to

$$
\sum_{l=1}^{(n / 2)-1} c_{l} c_{n-l} A_{l n}+d_{n} c_{n / 2}^{2}=0 \quad \text { for } n=4,6,8, \ldots
$$


and

$$
\sum_{l=1}^{(n-1) / 2} c_{l} c_{n-l} A_{l n}=0 \quad \text { for } n=3,5,7, \ldots
$$

Denote by $e_{n}=A_{1 n}=7+9 \times 3^{n}-14 \times 2^{n}-2 \times 4^{n}$. $e_{n}$ satisfies the relation

$$
e_{n+1}=-14+3 e_{n}+2^{n+1}\left(7-2^{n}\right) \text {. }
$$

Since $e_{0}=0$, we can compute the first few terms of this sequence. This yields $e_{1}=-2$, $e_{2}=0, e_{3}=10$ and $e_{4}=0$. Since $7-2^{n} \leqslant 0$ for $n \geqslant 3$, and $e_{4}=0,(45)$ implies $e_{n}<0$ for all $n \geqslant 5$.

For $n=3$, equation (44) is $c_{1} c_{2} e_{3}=0$. Since $e_{3}=10 \neq 0$, and $c_{1} \neq 0, c_{2}=0$. Since $e_{n} \neq 0$ for $n \geqslant 5$ we conclude inductively from (44) that $c_{n}=0$ for all even $n$. For $n=4$, equation (43) is satisfied identically, no matter the value of $c_{3}$, since $c_{2}=e_{4}=0$. Thus, the value of $c_{3}$ is not determined by the recursion relation (43). We will, finally, consider the two possibilities, $c_{3}=0$ or $c_{3} \neq 0$, separately. We need only consider equation (43), which takes the generic form

$$
c_{1} s_{k} e_{2 k}=g_{k}\left(s_{2}, s_{3}, \ldots, s_{k-1}\right) \quad \text { for } k=3,4,5, \ldots
$$

Here, we have introduced the notation $s_{k} \equiv c_{2 k-1}$ for $k=2,3, \ldots$ The functions $g_{k}$, on the right-hand side of (46), are homogeneous of degree 2 with respect to their arguments. If $s_{2} \equiv c_{3}=0$, we conclude inductively from (46) that $s_{k}=0$ for all $k \geqslant 2$, since $c_{1} \neq 0$ and $e_{2 k} \neq 0$ for $k \geqslant 3$. Therefore, if $c_{3}=0$, the analytic solution $\hat{g}(q)$ to equation (26) takes the form

$$
\hat{g}(q)=c_{1} q
$$

which is the symbol associated with the Korteweg-de Vries equation in equation (1).

To conclude our analysis, given $s_{2} \equiv c_{3} \neq 0$, equation (46) yields a well defined sequence $c_{3}, c_{5}, \ldots$ (since $c_{1} \neq 0$ and $e_{2 k} \neq 0$ for $k \geqslant 3$ ), where $c_{5}, c_{7}, \ldots$, are uniquely determined in terms of $c_{3}$. Since the solution to the recursion relation (given by equation (46)) is unique, and since we already know that

$$
\hat{g}(q)=\beta\left(\operatorname{coth}(\alpha q)-\frac{1}{\alpha q}\right)
$$

is an analytic solution to equation (26) with $c_{1}=\frac{1}{3} \alpha \beta \neq 0$ and $c_{3}=-\frac{1}{45} \alpha^{3} \beta \neq 0$, this is the only analytic solution (having $c_{1} \neq 0 \neq c_{3}$ ). The solution $\hat{g}(q)$ given by equation $(48)$ is the symbol associated with the ILW equation in equation (1).

\section{Acknowledgments}

We wish to thank Fernando Echeverría for useful discussions. This work was partially supported by FONDECYT, through the project no $0355-88$, and by $P$ Universidad Católica (DIUC grant no 47/87). 


\section{References}

Ablowitz M J, Fokas A S, Satsuma J and Segur H 1982 J. Phys. A: Math. Gen. 15781 Benjamin T B 1967 J. Fluid Mech. 29559

Case K 1979 J. Math. Phys. 20972

Faddeev L and Zakharov V E 1971 Funktional. Anal. i Prilozhen. 518

Gardner C S 1971 J. Math. Phys. 121548

Joseph R I 1977 J. Phys. A: Math. Gen. 11367

Kubota T, Ko D R S and Dobbs D 1978 J. Hydronaut. 12157

Lax P D 1975 Commun. Pure Appl. Math. 28141

Miura R, Gardner C S and Kruskal M D 1968 J. Math. Phys. 91204

Ono H 1975 J. Phys. Soc. Japan 391082

Satsuma J, Ablowitz M J and Kodama Y 1979 Phys. Lett. 73A 283 